\title{
Book Review - Neurofeedback in the Treatment of Developmental Trauma: Calming the Fear-Driven Brain
}

\author{
by Sebern F. Fisher. W. W. Norton \& Company, New York, NY, 2014, 416 pages, ISBN: 978-0-393-70786-1.
}

Citation: Jones, M. (2015). [Review of the book Neurofeedback in the Treatment of Developmental Trauma: Calming the Fear-Driven Brain, by Sebern F. Fisher]. NeuroRegulation, 2(2), 103-105. http://dx.doi.org/10.15540/nr.2.2.103

*Address correspondence to: Dr. Mark S. Jones, University of Texas at San Antonio, Department of Counseling, 501 W. Cesar E. Chavez Blvd, Durango Building 3.304, San Antonio, TX 78207-4415, USA. Email: mark.jones@utsa.edu

Copyright: (c) 2015. Jones. This is an Open Access article distributed under the terms of the Creative Commons Attribution License (CC-BY).
Edited by:

Nancy Wigton, PhD, Grand Canyon University, Arizona, USA

Reviewed by:

Randall Lyle, PhD, Mount Mercy University, lowa, USA
A long-awaited text from the originator of the FPO2 neurofeedback protocol for treating symptoms of psychological trauma, this book is both well crafted and beautifully written. Fisher gives us an insight into her own mind as she prosaically describes her clients from the framework of an experienced and compassionate clinician. The book thoroughly presents the treatment of fearful minds by bringing together psychotherapeutic theory, principles of EEG biofeedback, personal experience, and clear guidance.

The forward by Bessel van der Kolk, MD, sets the stage for the book's integration of developmental and psychological theory, neuroscience, and neurofeedback techniques. The structure of the book takes the reader first through foundational theory and developmental neuroanatomy, detailing the effects of trauma on the childhood brain and its subsequent insidious legacy for adulthood. Secondly, a primer on neurofeedback is woven into the various discussions of development, symptoms, and therapy, making the book a sound starting point for clinicians who are new to the field, as well as offering insights into the nuances of treatment that seasoned clinicians would appreciate. Numerous case studies shed light on the actual process of treatment and illustrate the heart-rending nature of working with this population.

In chapter 1, Fisher makes the case for a diagnostic category that transcends the limitations of two diagnoses commonly applied to persons with the symptoms she describes, namely posttraumatic stress disorder (PTSD) and reactive attachment disorder (RAD). Developmental trauma disorder (DTD) subsumes these two diagnoses into one cohesive syndrome. Fisher details the precursors of
DTD, which include attachment rupture, poverty, adverse events, and neglect. Symptomatically, DTD's hallmarks are aberrations in the capacity for empathy, and affective and sensory dysregulation. How these symptoms play out in life and therapy is further described, with special emphasis on the role of transference in the treatment process.

Chapters 2 and 3 comprise a thorough overview of the corpus of conventional neurofeedback concepts, with special attention and application to the treatment of DTD. Fisher goes into some depth on the neuroanatomical and neurophysiological effects of developmental trauma. Laying the groundwork for the reader's grasp of neurofeedback, she describes the significance of the various frequency bands and how EEG biofeedback is used in the amelioration of a dysregulated brain. For the potential client, this description gives a picture of what to expect in a treatment session. For clinicians acquainted with the arousal model as originally developed by the Othmers (Othmer, Othmer, \& Kaiser, 1999), this will be familiar territory.

Fisher's discussion of the nuts and bolts of neurofeedback is continued later in the book, but first she delves into the psychodynamics of developmental trauma in chapter 4, which is entitled "Trauma Identity." From the neuronal level to the complexities of the self, she covers a gamut of implications for trauma's effects against the framework of object relations and self-psychological concepts. It is this aspect of Fisher's writing that imparts a special dimension to her approach, which presumes that the treatment is done at the hands of clinicians who are not only skilled in the procedures of neurofeedback, but have a strong operational knowledge of the brain's functions and the mind's 
workings, and the developmental perspective to appreciate the encompassing process that therapy with these individuals entails.

Chapter 5 is a clinical guide to introducing clients to neurofeedback and the steps involved in forming the therapeutic relationship and beginning the assessment process. Early in the chapter Fisher emphasizes a symptom-based approach to protocol selection. Quantitative electroencephalogram (QEEG) analysis, in her practice, is reserved for clients with special issues and for cases in which treatment is not progressing as expected. Professional client-therapist issues are also discussed with specific recommendations related to this unique population and treatment approach.

Fisher places great importance in the book on assessment, which she covers in chapter 6 . Her approach provides the clinician with a clear path to selecting treatment protocols and a wealth of considerations befitting an encompassing model that bears out the deliberate long-term process of clinical experience, research, and collaboration that produced it. Fisher lays out a very systematic approach to assessing the client's symptoms and indications for specific treatment strategies. The initial assessment covers a variety of symptoms and presentation, including attention, sleep, emotional, behavioral, cognitive, pain, neurological, as well as autoimmune, autonomic nervous system, and endocrine issues-all of which are guiding factors in the arousal (and regulation) model. A plethora of additional considerations are discussed for gaining an even more complete picture of the client's condition. The ongoing assessment proceeds from session to session, and includes attention to the EEG itself and certain measures that may be applicable, as well as dealing with a plateau in progress and indications of when the therapeutic work is to be concluded.

Chapter 7 is a major expansion of the assessment process with in-depth descriptions of protocols. Various electrode placement sites, frequency bands to reward or inhibit, and the customization of protocols to suit the individual needs of each client are described with generous attention to clinical application and the rationale for each respective aspect. In combination with the foundational material presented earlier in the book, the discussion of treatment strategies takes on a particularly profound quality. Included in the protocol descriptions is a section devoted to the FPO2 protocol, which has historically been one of the most well-known features of the "Fisher protocol." Fisher based the protocol on a number of factors: the neurological concepts of Schore (1994) and LeDoux (1996), "as well as on traditions in body and energy work." At every turn, concepts are paired with clinical illustrations.

As the book contains a primer on neurofeedback, so it provides a primer on psychodynamic therapy, which is presented in chapter 8 . In so doing, Fisher continues her masterful work in integrating the two approaches into a cogent methodology. Basic psychodynamic concepts are explained and applied to the treatment of DTD, including transference and countertransference, regression, relationship development, shame, projection, the unconscious, dissociation, and finally, the emerging self structure.

The text of the entire book is amply illustrated with clinical examples. As if that isn't enough, Fisher provides a bonus feature at the end. Chapter 9 consists of detailed vignettes of three clients, including analyses of the treatment process for each.

Numerous tables and graphical illustrations are provided in the text, as well as a set of color figures in the book's center. Addenda contain Fisher's extensive Neurofeedback Assessment Questionnaire, a FAQ suitable for sharing with prospective clients, and a FPO2 Protocol Guide. A list of references and thorough index are provided.

In her book, Fisher offers much to readers in many ways. For those who specialize in the treatment of traumatized persons, this book is an indispensable guide even if the reader does not utilize neurofeedback! The discussion of human development and the neuroscience underlying developmental trauma stands on its own. For neurofeedback clinicians at any level of expertise, Fisher's writings are a comprehensive guide. For parents and those suffering from developmental trauma, here is a treasure trove of wisdom. In all, Fisher provides a validation to those who have worked with this population and a hopeful vision for the healing of these profound wounds.

Mark S. Jones, DMin, LPC-S, LMFT-S, BCN, QEEGD

Department of Counseling, University of Texas at San Antonio, San Antonio, Texas, USA 
Mark S. Jones, PLLC,

San Antonio, Texas, USA

Email: mark.jones@utsa.edu

\section{References}

LeDoux, J. E. (1996). The emotional brain: The mysterious underpinnings of emotional life. New York: Simon \& Schuster.

Othmer, S., Othmer, S. F., \& Kaiser, D. (1999). EEG Biofeedback: An Emerging Model for Its Global Efficacy. In J. R. Evans \& A. Abarbanel (Eds.), Introduction to Quantitative EEG and Neurofeedback (pp. 243-304). San Diego, CA: Academic Press.

Schore, A. N. (1994). Affect regulation and the origin of the self: The neurobiology of emotional development. Hillsdale, NJ: Lawrence Erlbaum Associates.

Received: June 27, 2015

Accepted: July 3, 2015

Published: July 15, 2015 